\title{
GENE EFFECTS AND INHERITANCE OF BRANCHING AND OTHER YIELD ATTRIBUTING CHARACTERISTICS IN SESAME (SESAMUM INDI- CUM L)
}

\author{
Sumathi $\mathrm{P}^{*}$ and Muralidharan V \\ Centre for Plant breeding and Genetics, Tamil Nadu Agricultural University, Coimbatore-641003, India
}

Accepted: 18 March 2014

\begin{abstract}
A field experiment was conducted with five sesame genotypes, two branched, TMV 4 and TMV 5, and three monostemed / shybranched KS 990837 , KS $990813 \mathrm{KS} 9908513$ and six cross combinations of branched $x$ monostemed to develop $\mathrm{F}_{1}, \mathrm{~F}_{2}, \mathrm{BC}_{1}$ and $\mathrm{BC}_{2}$ populations. Six generations $\mathrm{P}_{1}, \mathrm{P}_{2}, \mathrm{~F}_{1}, \mathrm{~F}_{2}, \mathrm{BC}_{1}$ and $\mathrm{BC}_{2}$ were raised in a non replicated trial to estimate the gene effects for branching and some other yield attributing characteristics. The additive, dominance and duplicate dominance gene effects were found to be important for number of branches per plant. Additive component was significantly positive for days to first flowering, days to maturity and plant height in TMV 4 x KS 99037; plant height and number of branches in TMV 5 x KS 990813; days to first flowering and days to maturity in the cross TMV $5 \times$ KS 99153; indicating the presence of additive gene action. Additiive $x$ additive fixable gene interaction was also reported in different crosses. In most of the crosses, duplicate epistasis was found to be predominant when compared to complementary epistasis. Thus gene effects for a given characteristics varied among crosses. Inheritance study using five crosses viz., TMV 4 x KS 99037, TMV 4 x KS 990813, TMV 5 x KS 99037, TMV 5 x KS990813 and TMV 5 x KS99153, showed a monogenic inheritance of 3:1 with simple dominance recessive relationship for branching and monostem / shy branching characteristic, respectively. The cross TMV4 x KS 99153 which showed 15:1 ratio, fitted to the expected ratio of 3:1 for branching : shybranching respectively of the test cross progenies. This cross also showed duplicate epistasis for the number of branches per plant in generation mean analysis.
\end{abstract}

Key words: Sesame, Monostem/shy branching, Gene action, Inheritance and epistasis

\section{INTRODUCTION}

Sesame (Sesamum indicum L.), is an annual plant, which is grown under tropical conditions. It is resistant to drought, short in maturity duration and suitable for wide range of cropping systems. Sesame seed is an important source of edible oil and is also widely used as a spice because of its ease of extraction and its great stability. Though the progress achieved in sesame in terms of cultivated area and production is quite high, its productivity in India is the lowest when compared to the world average. With our ever-increasing vegetable oil needs, it is time for us to take a second look at this ancient, versatile crop (Lal and Rai,1991). There is an inherent lacuna in sesame, as expected yields are not achieved due to poor population density. The branched varieties need more space per plant and hence maintenance of population density is very difficult in the commercial planting. This also poses serious problems in the countries where mechanical harvesting is restored. To overcome this situation, identification of mono stem or shy branching type of sesame suited to high - density planting is an important strategy, which will benefit the farming community by increased net return.

Literature on the inheritance of branching in sesame is limited. Joshi (1961), and Weiss (1971 and 1983) reviewed the inheritance of branching in sesame. Brar and Ahuja (1979) reported that the branching and non branching trait are monogenically controlled. Monostem characteristic is controlled, by recessive genes. Baydar and Turgut (2000) observed 3: 1 segregation ratio for branching and non-branching types, respectively. Multiple pathways involving different yield contributing characteristics influence the seed yield of sesame. Hence, seed

*Corresponding author: sumivetri@yahoo.com 
yield can also be improved through improvement of yield contributing characteristics such as days to first flowering, days to maturity, plant height, number of primary branches, number of capsules per plant, capsule length, number of seeds per capsule and 100 seed weight. To develop high yielding genotypes, the knowledge of gene action and relative magnitude of additive and non - additive genetic variance in yield is a pre-requisite to design efficient breeding programmes. Thus the present investigation is an attempt to study the inheritance and nature of gene action of branching and other quantitative characteristics that are contributing to yield in sesame.

\section{MATERIALS AND METHODS}

The experimental material was consisting of five sesame genotypes viz., two high yielding branched types, TMV 4 and TMV 5 and three monostem types, KS 99037, KS 990813 and KS 990153. Generation mean experiment was conducted using these five genotypes. Each of two branching types (TMV 4 and TMV 5) were crossed to each of three monostem types (KS 99037, KS 990813 and KS 990153) to get six combinations viz., TMV 4 x KS 99037, TMV 4 x KS 990813, TMV 4 x KS 990513, TMV 5 x KS 99037, TMV 5 x KS 990813 and TMV 5 x KS 990513. The $\mathrm{F}_{1}$ plants were randomly chosen from each cross and backcrossed to both the parents to produce $\mathrm{BC}_{1}\left(\mathrm{~F}_{1} \times \mathrm{P}_{1}\right)$ and $\mathrm{BC}_{2}\left(\mathrm{~F}_{1} \times \mathrm{P}_{2}\right)$ progenies. Fresh crosses were also made to produce fresh $F_{1}$ seeds. $F_{1}$ plants were selfed to produce $F_{2}$ generation. Thus, besides $\mathrm{P}_{1}$ and $\mathrm{P}_{2}$, seeds of four generations viz., $\mathrm{F}_{1}, \mathrm{~F}_{2}, \mathrm{BC}_{1}$ and $\mathrm{BC}_{2}$ were generated in each cross for generation mean analysis.

A field trial with six generations $\mathrm{P}_{1}, \mathrm{P}_{2}, \mathrm{~F}_{1}, \mathrm{~F}_{2}$, $\mathrm{BC}_{1}$ and $\mathrm{BC}_{2}$ was raised during rabi 2005-2006 at the Department of Oilseeds, Tamil Nadu Agricultural University, Coimbatore. The parents and $F_{1}$ s were planted in five rows of $5 \mathrm{~m}$ length. The $\mathrm{BC}_{1}$ and $\mathrm{BC}_{2}$ were planted in 5 rows of $6 \mathrm{~m}$ length, while the $\mathrm{F}_{2} \mathrm{~S}$ of different crosses were raised in 10 rows of $6 \mathrm{~m}$ length. The inter and intra row spacing was maintained at $30 \times 30 \mathrm{~cm}$. Standard cultivation practices were followed as per the recommendation. Seventy five plants from each of parents and $\mathrm{F}_{1} \mathrm{~s}, 100$ plants from each of $\mathrm{BC} 1$ and $\mathrm{BC}_{2}$ generations and 300 plants from each of $\mathrm{F}_{2}$ populations were randomly selected. Observations were recorded for all these selected individuals on days to first flowering, days to maturity, plant height, number of primary branches, number of capsules per plant, capsule length, number of seeds per capsule, 100 seed weight and seed yield per plant. The concept of generation mean analysis was developed by Hayman (1958) and Jinks and Jones (1958) for the estimation of genetic components of variation. Analysis of this technique is based on different generations of a cross viz., parents, their $\mathrm{F}_{1}, \mathrm{~F}_{2}$, and different backcrosses. The adequacy of the data for a simple additive-dominance model in a generation was first tested utilizing A, B and C scaling tests of Mather and Jinks (1971). The results obtained from the scaling tests were further confirmed by Joint scaling test as proposed by Cavalli (1952).

Inheritance studies: $\mathrm{Six}$ generations viz., $\mathrm{P}_{1}$, $\mathrm{P}_{2}, \mathrm{~F}_{1}, \mathrm{~F}_{2}, \mathrm{BC}_{1}$, and $\mathrm{BC}_{2}$ of the six cross combinations were used to study the mode of inheritance of branching. The individuals were grouped as branched ( $>2$ branches) and monostem / shy branching plants (0 to 2 branches) based on the number of branches per plant. The goodness of fit to Mendelian segregation ratios of branched and monostem / shy branched classes in the segregating population were tested by Chi- Square $\left(\mathrm{c}^{2}\right)$ test.

\section{RESULTS AND DISCUSSION}

Mean performance of six generation for various characteristics of different crosses in sesame are presented in Table 1. Among the parents considered in the study, TMV5 recorded the highest mean values for plant height, number of branches, number of capsules per plant and seed yield per plant (Table 1). The parent KS 99037 recorded the maximum number of seeds per capsule, while the minimum number was observed in the parents TMV 5 and TMV 4 with branching. 
Two hybrids viz., TMV 4 x KS 990813 and TMV 4 x KS 99037, exceeded its parents for number of capsules per plant and TMV $4 \mathrm{x}$ KS 99037 exceeded its parents for seed yield per plant and revealed over dominance. All the crosses exhibited higher number of branches per plant than that of their respective parent with lower number of branches. The hybrid TMV 4 x KS 990813 was early in duration and this hybrid exceeded in plant height when compared to its parents.
A comparison of the mean of $\mathrm{BC}_{1}$ with the mean of respective $F_{1} \mathrm{~s}$ revealed that only three back crosses viz., (TMV4 x KS 99037) $\mathrm{x}$ TMV4, (TMV4 x KS 99153) x TMV4, (TMV5 x KS 99153) x TMV5 recorded substantial increase in seed yield. The same back cross populations also recorded the highest increase in capsule number per plant. The comparison of $\mathrm{BC}_{2}$ population with $\mathrm{F}_{1}$ revealed that one $\mathrm{BC}_{2}$ population, (TMV5 $\mathrm{x} \mathrm{KS}$ 99153) x KS 99153 recorded the highest increase in capsule number and seed yield per plant.

Table 1. Mean performance of six generations for various characteristics of different crosses in sesame during rabi 2005-06 in Coimbatore, India

\begin{tabular}{|c|c|c|c|c|c|c|}
\hline \multirow[t]{2}{*}{ Cross } & \multicolumn{6}{|c|}{ Generation } \\
\hline & $\mathbf{P}_{1}$ & $\mathbf{P}_{2}$ & $\mathbf{F}_{1}$ & $\mathbf{F}_{2}$ & $\mathrm{BC}_{1}$ & $\mathrm{BC}_{2}$ \\
\hline \multicolumn{7}{|c|}{ Days to first flowering } \\
\hline TMV4 x KS99037 & $51.14^{1} \pm 0.22^{2}$ & $48.73 \pm 0.47$ & $47.03 \pm 0.67$ & $46.97 \pm 0.24$ & $49.6 \pm 0.34$ & $47.92 \pm 0.66$ \\
\hline TMV4 x KS990813 & $51.14 \pm 0.22$ & $42.23 \pm 0.20$ & $45.31 \pm 0.58$ & $48.41 \pm 0.23$ & $46.37 \pm 0.62$ & $46.59 \pm 0.58$ \\
\hline TMV4 x KS990153 & $51.14 \pm 0.22$ & $40.22 \pm 0.25$ & $45.26 \pm 0.70$ & $48.08 \pm 0.41$ & $45.73 \pm 0.89$ & $46.00 \pm 0.84$ \\
\hline TMV5 x KS99037 & $50.09 \pm 0.18$ & $48.73 \pm 0.47$ & $41.96 \pm 0.90$ & $46.82 \pm 0.32$ & $43.00 \pm 0.44$ & $44.84 \pm 0.76$ \\
\hline TMV5 x KS990813 & $50.09 \pm 0.18$ & $42.23 \pm 0.20$ & $39.95 \pm 0.41$ & $41.81 \pm 0.34$ & $41.50 \pm 0.58$ & $41.15 \pm 0.54$ \\
\hline TMV5 x KS990153 & $50.09 \pm 0.18$ & $40.22 \pm 0.25$ & $41.10 \pm 0.54$ & $42.24 \pm 0.36$ & $48.45 \pm 0.72$ & $43.29 \pm 0.93$ \\
\hline \multicolumn{7}{|l|}{ Days to maturity } \\
\hline TMV4 x KS99037 & $99.71 \pm 0.29$ & $95.79 \pm 0.81$ & $90.83 \pm 1.29$ & $93.47 \pm 0.48$ & $98.80 \pm 0.56$ & $95.46 \pm 1.25$ \\
\hline TMV4 x KS990813 & $99.71 \pm 0.29$ & $84.19 \pm 0.43$ & $91.09 \pm 1.05$ & $96.0 \pm 0.38$ & $92.83 \pm 1.30$ & $93.59 \pm 1.19$ \\
\hline TMV4 x KS990153 & $99.71 \pm 0.29$ & $80.57 \pm 0.35$ & $92.51 \pm 1.19$ & $96.22 \pm 0.71$ & $91.92 \pm 1.52$ & $92.50 \pm 1.65$ \\
\hline TMV5 x KS99037 & $100.01 \pm 0.23$ & $95.79 \pm 0.81$ & $84.57 \pm 1.66$ & $93.42 \pm 0.61$ & $86.04 \pm 0.92$ & $90.40 \pm 1.48$ \\
\hline TMV5 x KS990813 & $100.01 \pm 0.23$ & $84.19 \pm 0.43$ & $80.12 \pm 0.66$ & $84.26 \pm 0.60$ & $83.27 \pm 1.07$ & $83.62 \pm 1.06$ \\
\hline TMV5 x KS990153 & $100.01 \pm 0.23$ & $80.57 \pm 0.35$ & $82.94 \pm 0.99$ & $84.74 \pm 0.68$ & $96.73 \pm 1.36$ & $86.71 \pm 1.79$ \\
\hline \multicolumn{7}{|l|}{ Plant height } \\
\hline TMV4 x KS99037 & $94.35 \pm 1.57$ & $89.87 \pm 2.50$ & $91.08 \pm 2.58$ & $90.13 \pm 0.90$ & $108.30 \pm 2.95$ & $95.19 \pm 3.05$ \\
\hline TMV4 x KS990813 & $94.35 \pm 1.57$ & $71.41 \pm 1.78$ & $103.47 \pm 2.91$ & $96.21 \pm 1.17$ & $74.83 \pm 3.07$ & $91.05 \pm 2.34$ \\
\hline TMV4 x KS990153 & $94.35 \pm 1.57$ & $62.01 \pm 1.98$ & $88.57 \pm 2.55$ & $97.52 \pm 1.56$ & $106.0 \pm 3.88$ & $96.61 \pm 4.28$ \\
\hline TMV5 x KS99037 & $106.91 \pm 1.85$ & $89.87 \pm 2.50$ & $87.43 \pm 3.23$ & $86.45 \pm 1.55$ & $89.5 \pm 2.22$ & $90.66 \pm 3.11$ \\
\hline TMV5 x KS990813 & $106.91 \pm 1.85$ & $71.41 \pm 1.78$ & $96.45 \pm 1.72$ & $87.25 \pm 1.70$ & $81.68 \pm 2.39$ & $69.88 \pm 3.15$ \\
\hline TMV5 x KS990153 & $106.91 \pm 1.85$ & $62.01 \pm 1.98$ & $83.36 \pm 2.83$ & $82.78 \pm 1.82$ & $94.86 \pm 3.10$ & $93.48 \pm 4.08$ \\
\hline \multicolumn{7}{|l|}{ No. of branches } \\
\hline TMV4 x KS99037 & $4.38 \pm 0.15$ & $2.07 \pm 0.17$ & $4.05 \pm 0.31$ & $3.99 \pm 0.12$ & $4.35 \pm 0.41$ & $4.24 \pm 0.40$ \\
\hline TMV4 x KS990813 & $4.38 \pm 0.15$ & $2.24 \pm 0.15$ & $4.64 \pm 0.32$ & $4.29 \pm 0.16$ & $3.72 \pm 0.35$ & $3.73 \pm 0.27$ \\
\hline TMV4 x KS990153 & $4.38 \pm 0.15$ & $1.81 \pm 0.15$ & $5.00 \pm 0.28$ & $4.95 \pm 0.17$ & $4.51 \pm 0.40$ & $4.41 \pm 0.49$ \\
\hline TMV5 x KS99037 & $5.41 \pm 0.20$ & $2.07 \pm 0.17$ & $4.27 \pm 0.26$ & $3.88 \pm 0.14$ & $4.14 \pm 0.21$ & $3.49 \pm 0.29$ \\
\hline TMV5 x KS990813 & $5.41 \pm 0.20$ & $2.24 \pm 0.15$ & $4.69 \pm 0.24$ & $3.43 \pm 0.15$ & $3.59 \pm 0.17$ & $2.96 \pm 0.25$ \\
\hline TMV5 x KS990153 & $5.41 \pm 0.20$ & $1.81 \pm 0.15$ & $4.39 \pm 0.29$ & $4.12 \pm 0.12$ & $4.69 \pm 0.38$ & $3.72 \pm 0.37$ \\
\hline \multicolumn{7}{|c|}{ No. capsules per plant } \\
\hline TMV4 x KS99037 & $65.29 \pm 3.61$ & $48.96 \pm 3.44$ & $79.75 \pm 5.44$ & $48.86 \pm 1.88$ & $104.4 \pm 6.94$ & $79.81 \pm 5.73$ \\
\hline TMV4 x KS990813 & $65.29 \pm 3.61$ & $41.63 \pm 2.29$ & $81.47 \pm 5.63$ & $68.43 \pm 2.48$ & $62.77 \pm 5.64$ & $72.27 \pm 3.99$ \\
\hline TMV4 x KS990153 & $65.29 \pm 3.61$ & $47.12 \pm 3.22$ & $65.70 \pm 4.33$ & $62.21 \pm 2.94$ & $80.35 \pm 5.68$ & $65.07 \pm 5.72$ \\
\hline
\end{tabular}


SUMATHI AND MURALIDHARAN : INHERITANCE FOR BRANCHING IN SESAME

\begin{tabular}{|c|c|c|c|c|c|c|}
\hline TMV5 x KS99037 & $77.27 \pm 3.52$ & $48.96 \pm 3.44$ & $86.70 \pm 6.07$ & $60.66 \pm 2.52$ & $64.66 \pm 4.42$ & $82.78 \pm 6.31$ \\
\hline TMV5 x KS990813 & $77.27 \pm 3.52$ & $41.63 \pm 2.29$ & $63.02 \pm 4.63$ & $64.70 \pm 2.51$ & $51.79 \pm 3.41$ & $54.50 \pm 5.15$ \\
\hline TMV5 x KS990153 & $77.27 \pm 3.52$ & $47.12 \pm 3.22$ & $57.46 \pm 4.31$ & $68.41 \pm 3.18$ & $82.59 \pm 4.75$ & $88.10 \pm 6.54$ \\
\hline \multicolumn{7}{|l|}{ Capsule length (cm) } \\
\hline TMV4 x KS99037 & $2.48 \pm 0.03$ & $2.82 \pm 0.03$ & $2.58 \pm 0.05$ & $2.62 \pm 0.02$ & $2.67 \pm 0.06$ & $2.65 \pm 0.05$ \\
\hline TMV4 x KS990813 & $2.48 \pm 0.03$ & $2.59 \pm 0.03$ & $2.59 \pm 0.04$ & $2.61 \pm 0.02$ & $2.60 \pm 0.04$ & $2.75 \pm 0.05$ \\
\hline TMV4 x KS990153 & $2.48 \pm 0.03$ & $2.70 \pm 0.03$ & $2.45 \pm 0.04$ & $2.51 \pm 0.02$ & $2.63 \pm 0.06$ & $2.75 \pm 0.04$ \\
\hline TMV5 x KS99037 & $2.50 \pm 0.03$ & $2.82 \pm 0.03$ & $2.69 \pm 0.04$ & $2.62 \pm 0.02$ & $2.65 \pm 0.03$ & $2.59 \pm 0.04$ \\
\hline TMV5 x KS990813 & $2.50 \pm 0.03$ & $2.59 \pm 0.03$ & $2.46 \pm 0.04$ & $2.62 \pm 0.02$ & $2.58 \pm 0.03$ & $2.80 \pm 0.03$ \\
\hline TMV5 x KS990153 & $2.50 \pm 0.03$ & $2.70 \pm 0.03$ & $2.43 \pm 0.03$ & $2.42 \pm 0.02$ & $2.87 \pm 0.05$ & $3.04 \pm 0.07$ \\
\hline \multicolumn{7}{|c|}{ No. of seeds per capsule } \\
\hline TMV4 x KS99037 & $44.82 \pm 0.82$ & $58.75 \pm 0.64$ & $51.19 \pm 1.61$ & $50.91 \pm 0.63$ & $52.02 \pm 2.46$ & $52.76 \pm 1.58$ \\
\hline TMV4 x KS990813 & $44.82 \pm 0.82$ & $51.37 \pm 0.97$ & $50.47 \pm 1.57$ & $49.60 \pm 0.69$ & $49.63 \pm 1.28$ & $56.83 \pm 1.96$ \\
\hline TMV4 x KS990153 & $44.82 \pm 0.82$ & $51.40 \pm 0.87$ & $46.00 \pm 1.47$ & $45.07 \pm 0.72$ & $49.52 \pm 1.65$ & $52.17 \pm 1.32$ \\
\hline TMV5 x KS99037 & $43.48 \pm 0.98$ & $58.75 \pm 0.64$ & $53.16 \pm 1.20$ & $48.82 \pm 0.58$ & $48.09 \pm 1.09$ & $47.90 \pm 1.26$ \\
\hline TMV5 x KS990813 & $43.48 \pm 0.98$ & $51.37 \pm 0.97$ & $44.63 \pm 1.19$ & $46.09 \pm 0.81$ & $47.38 \pm 0.95$ & $53.28 \pm 1.40$ \\
\hline TMV5 x KS990153 & $43.48 \pm 0.98$ & $51.40 \pm 0.87$ & $42.03 \pm 1.54$ & $43.34 \pm 0.88$ & $54.39 \pm 1.12$ & $62.42 \pm 0.86$ \\
\hline \multicolumn{7}{|l|}{100 seed weight $(g)$} \\
\hline TMV4 x KS99037 & $0.33 \pm 0.006$ & $0.28 \pm 0.007$ & $0.29 \pm 0.010$ & $0.29 \pm 0.003$ & $0.32 \pm 0.01$ & $0.32 \pm 0.009$ \\
\hline TMV4 x KS990813 & $0.33 \pm 0.006$ & $0.28 \pm 0.004$ & $0.30 \pm 0.010$ & $0.31 \pm 0.004$ & $0.30 \pm 0.007$ & $0.29 \pm 0.007$ \\
\hline TMV4 x KS990153 & $0.33 \pm 0.006$ & $0.27 \pm 0.004$ & $0.29 \pm 0.007$ & $0.29 \pm 0.005$ & $0.29 \pm 0.011$ & $0.28 \pm 0.008$ \\
\hline TMV5 x KS99037 & $0.33 \pm 0.005$ & $0.28 \pm 0.007$ & $0.29 \pm 0.010$ & $0.29 \pm 0.003$ & $0.27 \pm 0.005$ & $0.31 \pm 0.009$ \\
\hline TMV5 x KS990813 & $0.33 \pm 0.005$ & $0.28 \pm 0.004$ & $0.30 \pm 0.007$ & $0.30 \pm 0.003$ & $0.30 \pm 0.006$ & $0.29 \pm 0.007$ \\
\hline TMV5 x KS990153 & $0.33 \pm 0.005$ & $0.27 \pm 0.004$ & $0.29 \pm 0.007$ & $0.28 \pm 0.004$ & $0.31 \pm 0.10$ & $0.31 \pm 0.10$ \\
\hline \multicolumn{7}{|c|}{ Seed yield per plant (g) } \\
\hline TMV4 x KS99037 & $7.91 \pm 0.54$ & $4.89 \pm 0.45$ & $9.32 \pm 1.06$ & $4.53 \pm 0.20$ & $11.31 \pm 1.62$ & $8.65 \pm 0.78$ \\
\hline TMV4 x KS990813 & $7.91 \pm 0.54$ & $3.50 \pm 0.22$ & $8.65 \pm 0.92$ & $7.28 \pm 0.34$ & $5.94 \pm 0.68$ & $7.60 \pm 0.64$ \\
\hline TMV4 x KS990153 & $7.91 \pm 0.54$ & $3.63 \pm 0.43$ & $6.67 \pm 0.62$ & $5.99 \pm 0.39$ & $8.52 \pm 1.23$ & $6.34 \pm 0.82$ \\
\hline TMV5 x KS99037 & $8.64 \pm 0.47$ & $4.89 \pm 0.45$ & $8.81 \pm 0.94$ & $5.16 \pm 0.26$ & $5.56 \pm 0.50$ & $9.52 \pm 1.11$ \\
\hline TMV5 x KS990813 & $8.64 \pm 0.47$ & $3.50 \pm 0.22$ & $6.68 \pm 0.71$ & $6.23 \pm 0.33$ & $4.93 \pm 0.42$ & $5.38 \pm 0.74$ \\
\hline MV5 x KS990153 & $8.64 \pm 0.47$ & $3.63 \pm 0.43$ & $6.09 \pm 0.61$ & $6.76 \pm 0.50$ & $8.97 \pm 1.09$ & $11.13 \pm 1.49$ \\
\hline \multicolumn{7}{|l|}{ Oil content $(\%)$} \\
\hline TMV4 x KS99037 & $40.72 \pm 0.16$ & $39.78 \pm 0.13$ & $40.92 \pm 0.15$ & $39.78 \pm 0.09$ & $40.74 \pm 0.26$ & $41.00 \pm 0.23$ \\
\hline TMV4 x KS990813 & $40.72 \pm 0.16$ & $39.69 \pm 0.15$ & $40.19 \pm 0.28$ & $40.07 \pm 0.12$ & $40.79 \pm 0.37$ & $41.50 \pm 0.30$ \\
\hline TMV4 x KS990153 & $40.72 \pm 0.16$ & $39.23 \pm 0.16$ & $40.01 \pm 0.20$ & $40.12 \pm 0.15$ & $39.72 \pm 0.31$ & $40.43 \pm 0.25$ \\
\hline TMV5 x KS99037 & $39.92 \pm 0.18$ & $39.78 \pm 0.13$ & $41.78 \pm 0.38$ & $40.11 \pm 0.10$ & $40.02 \pm 0.21$ & $40.06 \pm 0.21$ \\
\hline TMV5 x KS990813 & $39.92 \pm 0.18$ & $39.69 \pm 0.15$ & $40.27 \pm 0.21$ & $40.05 \pm 0.14$ & $40.07 \pm 0.19$ & $40.04 \pm 0.20$ \\
\hline TMV5 x KS990153 & $39.92 \pm 0.18$ & $39.23 \pm 0.16$ & $39.64 \pm 0.19$ & $39.86 \pm 0.12$ & $39.82 \pm 0.31$ & $39.99 \pm 0.29$ \\
\hline
\end{tabular}

$1 \mathbf{P}_{1}-$ Parent 1(Female), $\mathbf{P}_{2}-$ Parent 2 (Male), $\mathbf{F}_{1}-$ First filial generation, $\mathbf{F}_{2}-$ Second filial generation, $\mathbf{B C}_{1}-$ Back cross 1, $\mathrm{BC}_{2}-$ Back cross 2,

2 Values with $\pm \mathrm{SE}$

Gene effects for a given characteristic varied among crosses. Thus, the simple additivedominance model was not adequate to explain the inheritance of all the characteristics studied (Table 2) in all the cross combinations except in TMV5 x KS 99153 for number of branches in TMV 4 x KS 99037, for capsule length and number of seeds per capsule, TMV 4 x KS 990813, TMV 4 x KS 99153 and
TMV 5 x KS 990813 for 100-seed weight, TMV 4 x KS 99153 for number of capsules per plant and seed yield per plant and TMV5 x KS 990813 and TMV 5 x KS 99153, for oil content. The segregating generations of the above crosses are likely to yield promising progenies as they fit well with the simple additive-dominance model. Therefore, it is inferred that pedigree-breeding procedure is 
adequate for achieving the breeding objectives for improving the indicated economic characteristics using the above crosses. However, for the rest of the characters simple additive-dominance model was not adequate to explain the inheritance, using all the other remaining crosses, which showed the complexity of characteristics and hence the analysis was further extended to a digenic interaction.

Table 2. Genetic components estimated using GMA with 3 and 6 parameter models for different characteristics in sesame

\begin{tabular}{|c|c|c|c|c|c|c|c|c|c|c|}
\hline Crosses & $\mathbf{A}$ & B & $\mathbf{C}$ & $\mathbf{c}^{2}$ & {$[\mathbf{m}]$} & [d] & [h] & [i] & [j] & [1] \\
\hline \multicolumn{11}{|c|}{ Days to first flowering } \\
\hline TMV4 x KS99037 & 1.04 & 0.09 & $-6.06 * *$ & $27.48 * *$ & $46.97 * *$ & $1.68^{*}$ & $4.28 *$ & $7.18^{* *}$ & 0.47 & $-8.31 * *$ \\
\hline TMV4 x KS990813 & $-3.72 * *$ & $5.64 * *$ & $9.65^{* * *}$ & $74.78 * *$ & $48.41 * *$ & -0.22 & $-9.09 * *$ & $-7.73^{*}$ & $-4.68 *$ & 5.80 \\
\hline TMV4 x KS990153 & $-4.93^{*}$ & $6.53 * *$ & $10.45^{* *}$ & $45.21^{* *}$ & $48.08^{* *}$ & -0.27 & $-9.27 * *$ & $-8.85^{* *}$ & $-5.73 * *$ & 7.25 \\
\hline TMV5 x KS99037 & $-6.04 * *$ & -0.10 & $4.56^{* *}$ & $56.54 * *$ & $46.82 * *$ & $-1.84 *$ & $-19.06 * *$ & $-11.60 * *$ & $-2.52 * *$ & $18.65^{* *}$ \\
\hline TMV5 x KS990813 & $-7.04 * *$ & 0.12 & $-4.97 * *$ & $37.71 * *$ & $41.81 * *$ & 0.35 & $-8.16 * *$ & -1.96 & $-3.58 * *$ & $8.88^{*}$ \\
\hline TMV5 x KS990153 & $5.72 * *$ & $5.26 * *$ & -3.53 & $29.31 * *$ & $42.24 * *$ & $5.17 * *$ & $10.46 *$ & $14.51^{* *}$ & 0.23 & $-25.49 * *$ \\
\hline \multicolumn{11}{|c|}{ Days to maturity } \\
\hline TMV4 x KS99037 & $7.05 * *$ & 4.30 & -3.30 & $36.59 * *$ & $93.47 * *$ & $3.34 *$ & $7.74 *$ & $14.66^{* *}$ & 1.38 & $-26.01 * *$ \\
\hline TMV4 x KS990813 & -5.14 & $11.90 *$ & $20.32 *$ & $83.10^{* * *}$ & $96.60 * *$ & -0.76 & $-14.41 * *$ & $-13.56^{* *}$ & $-8.52 * *$ & 6.79 \\
\hline TMV4 x KS990153 & $-8.38^{*}$ & $11.92 * *$ & $19.57 * *$ & $49.29^{* *}$ & $96.22 * *$ & -0.58 & $-13.65^{*}$ & $-16.02 * *$ & $-10.15^{* *}$ & 12.48 \\
\hline TMV5 x KS99037 & $-12.50^{* *}$ & 0.46 & $8.76^{*}$ & $56.84^{* *}$ & $93.42 * *$ & $-4.37 *$ & $-34.14 * *$ & $-20.81 * *$ & $-6.48^{* *}$ & $32.85^{* *}$ \\
\hline TMV5 x KS990813 & $-13.60^{* *}$ & 2.93 & $-7.38 * *$ & 43.88 & $84.26 * *$ & -0.35 & $-15.27 * *$ & -3.29 & $-8.26 * *$ & $13.96^{* *}$ \\
\hline TMV5 x KS990153 & $10.50^{* *}$ & $9.92 * *$ & $-7.51 * *$ & $30.20^{* *}$ & $84.74 * *$ & $10.01 * *$ & $20.58 * *$ & $27.93 * *$ & 0.29 & $-48.35^{* *}$ \\
\hline \multicolumn{11}{|c|}{ Plant height } \\
\hline TMV4 x KS99037 & $31.17 * *$ & 9.44 & -5.86 & $32.10^{* *}$ & $90.13 * *$ & $13.11 * *$ & $45.44 * *$ & $46.46^{* *}$ & $10.87^{*}$ & $-87.06 * *$ \\
\hline TMV4 x KS990813 & $-48.15^{* *}$ & 7.21 & 12.15 & $67.07 * *$ & $96.21^{* *}$ & $-16.21^{* *}$ & $-32.51 * *$ & $-53.10 * *$ & $-27.68 * *$ & $94.04 * *$ \\
\hline TMV4 x KS990153 & $29.78^{* *}$ & $42.63 * *$ & $56.58 * *$ & $59.36^{* *}$ & $97.52 * *$ & 9.39 & 25.52 & 15.13 & $-6.77 *$ & $-86.83 * *$ \\
\hline TMV5 x KS99037 & $-15.33 * *$ & 4.01 & $-25.85^{* *}$ & 13.48 & $86.45^{* *}$ & -1.15 & 3.58 & 14.53 & $-9.67 *$ & -3.22 \\
\hline TMV5 x KS990813 & $-40.01 * *$ & $-28.10 * *$ & $-22.25^{* *}$ & $67.86^{* *}$ & $87.25^{* *}$ & $11.80 * *$ & $-38.57 * *$ & $-45.86 * *$ & -5.95 & $113.97^{* *}$ \\
\hline TMV5 x KS990153 & -0.55 & $41.58 * *$ & -4.54 & 24.71 & $82.78 * *$ & 1.39 & $44.47 * *$ & $45.58 * *$ & $-21.06^{* *}$ & $-86.61 * *$ \\
\hline \multicolumn{11}{|c|}{ No.of primary branches } \\
\hline TMV4 x KS99037 & 0.027 & $2.37 * *$ & 1.39 & 8.34 & $3.99 * *$ & 0.11 & 2.06 & $1.24 *$ & 1.05 & -3.87 \\
\hline TMV4 x KS990813 & $-1.59 *$ & 0.58 & 1.24 & $8.87 *$ & $4.29^{* *}$ & -0.02 & -0.91 & -2.25 & $-1.09^{*}$ & 3.26 \\
\hline TMV4 x KS990153 & -0.37 & 2.00 & $3.58 * *$ & $19.16^{* *}$ & $4.95^{* *}$ & 0.10 & -0.03 & -1.95 & -1.18 & 0.31 \\
\hline TMV5 x KS99037 & $-1.39 *$ & 0.64 & -0.49 & $8.75^{*}$ & $3.88 * *$ & 0.66 & 0.28 & -0.25 & $-1.01 *$ & 1.00 \\
\hline TMV5 x KS990813 & $-2.93 * *$ & -1.01 & $-3.28 * *$ & $43.18^{* *}$ & $3.44^{* *}$ & $0.62 *$ & 0.22 & -0.65 & $-0.96 * *$ & $4.59 *$ \\
\hline TMV5 x KS990153 & -0.42 & 1.24 & 0.51 & 2.99 & $4.12^{* *}$ & 0.97 & 1.09 & a & $\mathrm{a}$ & $\mathrm{a}$ \\
\hline \multicolumn{11}{|c|}{ No.of capsules per plant } \\
\hline TMV4 x KS99037 & $48.86^{* *}$ & $30.90 *$ & $-78.31 * *$ & $81.46^{* *}$ & $48.86^{* *}$ & 17.14 & $180.7 * *$ & $158.07 * *$ & 8.98 & $-237.84 * *$ \\
\hline TMV4 x KS990813 & -21.22 & $21.45^{*}$ & 3.86 & $9.43 *$ & $68.43^{* *}$ & -9.51 & 24.38 & -3.63 & $-21.34 * *$ & 3.40 \\
\hline TMV4 x KS990153 & $29.70^{*}$ & 17.32 & 5.02 & 7.00 & $62.21 * *$ & 15.27 & $51.50 *$ & a & $\mathrm{a}$ & $\mathrm{a}$ \\
\hline TMV5 x KS99037 & $-34.65 * *$ & $29.91 *$ & $-56.99 * *$ & $28.19^{* *}$ & $60.66 * *$ & $-18.12 *$ & $75.83^{* *}$ & $52.25 *$ & $-32.28 * *$ & -47.51 \\
\hline TMV5 x KS990813 & $-36.72 * *$ & 4.35 & 13.87 & $26.34 * *$ & $64.70 * *$ & -2.71 & $-42.67 *$ & $-46.24 *$ & $-20.54 * *$ & $78.42 * *$ \\
\hline TMV5 x KS990153 & $30.45^{* *}$ & $71.61 * *$ & $34.33 *$ & $30.69^{* *}$ & $68.41 * *$ & -5.50 & $62.99 * *$ & $67.73^{* *}$ & $-20.58^{*}$ & $-169.79^{* *}$ \\
\hline \multicolumn{11}{|c|}{ Capsule length } \\
\hline TMV4 x KS99037 & $0.28 *$ & -0.11 & 0.003 & 6.55 & $2.62 * *$ & 0.02 & 0.09 & a & a & a \\
\hline TMV4 x KS990813 & 0.12 & $0.31 * *$ & 0.17 & 8.89 & $2.61^{* *}$ & $-0.15^{*}$ & $0.32 *$ & 0.26 & -0.09 & $-0.69 *$ \\
\hline TMV4 x KS990153 & $0.32 *$ & $0.36 * *$ & -0.03 & $24.68 * *$ & $2.51 * *$ & -0.12 & $0.57 * *$ & $0.71^{* *} *$ & -0.02 & $-1.39 * *$ \\
\hline TMV5 x KS99037 & 0.11 & $-0.34 * *$ & $-0.25^{*}$ & $21.82 * *$ & $2.62 * *$ & 0.06 & 0.05 & 0.02 & $0.22 * *$ & 0.21 \\
\hline TMV5 x KS990813 & $0.21 * *$ & $0.55^{* *}$ & $0.48 * *$ & $52.31 * *$ & $2.62 * *$ & $-0.22 * *$ & 0.20 & $0.28 *$ & $0.17 * *$ & $-1.04 * *$ \\
\hline TMV5 x KS990153 & $0.81 * *$ & $0.96 * *$ & $-0.39 * *$ & $120.70^{* *}$ & $2.42 * *$ & $-0.17 *$ & $1.99 * *$ & $2.16^{* *}$ & -0.08 & $-3.93 * *$ \\
\hline \multicolumn{11}{|c|}{ No .of seeds per capsule } \\
\hline TMV4 x KS99037 & 8.04 & -4.42 & -2.29 & 4.72 & $50.91 * *$ & -0.74 & 5.31 & $\mathrm{a}$ & $\mathrm{a}$ & $\mathrm{a}$ \\
\hline TMV4 x KS990813 & 3.97 & $11.81^{* *}$ & 1.25 & $8.97 *$ & $45.60 * *$ & $-7.19^{* *}$ & $16.92 * *$ & $14.54 * *$ & -3.92 & $-30.32 * *$ \\
\hline TMV4 x KS990153 & $8.22 *$ & $6.94 *$ & -7.94 & $20.55 * *$ & $45.07 * *$ & -2.65 & $20.99 * *$ & $23.11^{* *}$ & 0.64 & $-38.27 * *$ \\
\hline TMV5 x KS99037 & -0.45 & $-16.10 * *$ & $-13.27 * *$ & $39.07 * *$ & $48.82 * *$ & 0.19 & -1.25 & -3.28 & $7.83 * *$ & $19.83 *$ \\
\hline TMV5 x KS990813 & $6.64 * *$ & $10.55^{* *}$ & 0.25 & $18.43 * *$ & $46.09 * *$ & $-5.90 * *$ & $14.15^{* *}$ & $16.94 * *$ & -1.96 & $-34.14 * *$ \\
\hline TMV5 x KS990153 & $23.26^{* *}$ & $31.42 * *$ & -5.59 & $269.24 * *$ & & $43.34 * *$ & $-8.04 * *$ & $54.86^{* *}$ & $60.28 * *$ & $-4.08 * *$ \\
\hline \multicolumn{11}{|c|}{100 seed weight } \\
\hline TMV4 x KS99037 & 0.009 & $0.06 * *$ & -0.040 & $19.79 * *$ & $0.29^{* *}$ & -0.001 & $0.103 * *$ & $0.11^{*}$ & -0.03 & $-0.18^{*}$ \\
\hline TMV4 x KS990813 & -0.031 & 0.007 & 0.004 & 4.05 & $0.31 * *$ & 0.010 & -0.04 & a & $\mathrm{a}$ & $\mathrm{a}$ \\
\hline TMV4 x KS990153 & -0.04 & -0.003 & 0.008 & 2.77 & $0.29 * *$ & 0.01 & -0.06 & a & $\mathrm{a}$ & $\mathrm{a}$ \\
\hline TMV5 x KS99037 & $-0.08^{* *}$ & $0.05^{*}$ & -0.04 & $44.97 * *$ & $0.29 * *$ & $-0.04 * *$ & -0.003 & 0.007 & $-0.06 * *$ & 0.03 \\
\hline TMV5 x KS990813 & $-0.04 *$ & -0.005 & -0.021 & 6.01 & $0.30 * *$ & 0.006 & -0.03 & $\mathrm{a}$ & $\mathrm{a}$ & $\mathrm{a}$ \\
\hline TMV5 x KS990153 & -0.002 & $0.067^{* *}$ & $-0 . .068 * *$ & $24.74 * *$ & $0.28 * *$ & -0.005 & $0.13 * *$ & $0.13 * *$ & $0.03 *$ & $-0.20 * *$ \\
\hline \multicolumn{11}{|c|}{ Seed yield per plant } \\
\hline TMV4 x KS99037 & 5.39 & 3.09 & $-13.32 * *$ & $72.91 * *$ & $4.53^{* *}$ & 2.66 & $24.71 * *$ & $21.79^{* *}$ & 1.15 & $-30.26 * *$ \\
\hline TMV4 x KS990813 & $-4.68 * *$ & 3.06 & 0.43 & $16.19^{* *}$ & $7.28 * *$ & -1.67 & 0.90 & -2.05 & $-3.87 * *$ & 3.67 \\
\hline
\end{tabular}




\begin{tabular}{|c|c|c|c|c|c|c|c|c|c|c|}
\hline TMV4 x KS990153 & 2.47 & 2.38 & -0.92 & 3.36 & $5.99 * *$ & 2.18 & 6.67 & $\bar{a}$ & $\bar{a}$ & $\mathrm{a}$ \\
\hline TMV5 x KS99037 & $-6.33 * *$ & $5.34 *$ & $-10.52 * *$ & $43.44 * *$ & $5.16 * *$ & $-3.96 * *$ & $11.57 * *$ & $9.52 * *$ & $-5.83 * *$ & -8.54 \\
\hline TMV5 x KS990813 & $-5.47 * *$ & 0.57 & 0.57 & $28.01 * *$ & $6.23 * *$ & -0.45 & -3.71 & $-4.32 *$ & $-3.02 * *$ & $9.22 *$ \\
\hline TMV5 x KS990153 & 3.22 & $12.54 * *$ & 2.58 & $18.17 * *$ & $6.76 * *$ & -2.16 & $13.14 * *$ & $13.18 * *$ & $-4.66^{*}$ & $28.94 * *$ \\
\hline \multicolumn{11}{|c|}{ Oil content } \\
\hline TMV4 x KS99037 & -0.16 & $1.31 * *$ & $-3.23 * *$ & $56.88 * *$ & $39.78 * *$ & -0.27 & $5.05^{*}$ & $4.38^{* * *}$ & $-0.74 *$ & $-5.52 * *$ \\
\hline TMV4 x KS990813 & 0.67 & $3.12 * *$ & -0.50 & $27.42 * *$ & $40.07 * *$ & -0.71 & $4.27 * *$ & $4.29 * *$ & $-1.23^{*}$ & $-8.08 * *$ \\
\hline TMV4 x KS990153 & -1.29 & $1.63 * *$ & 0.52 & 13.50 & $40.12 * *$ & -0.71 & $-0.15^{*}$ & -0.18 & -1.46 & -0.17 \\
\hline TMV5 x KS99037 & $-1.67 * *$ & $-1.43 *$ & $-2.84 * *$ & $11.98 * *$ & 40.11 & -0.05 & $1.67^{*}$ & -0.26 & -0.12 & $3.36^{*}$ \\
\hline TMV5 x KS990813 & -0.05 & 0.12 & 0.04 & 0,09 & $40.05 * *$ & 0.03 & 0.49 & $\mathrm{a}$ & $\mathrm{a}$ & a \\
\hline TMV5 x KS990153 & 0.08 & 1.11 & 0.10 & 4.51 & $39.86 * *$ & -0.17 & 0.26 & $\mathrm{a}$ & a & a \\
\hline
\end{tabular}

*Significant at $5 \%$ level **Significant at $1 \%$ level a -Adequacy of additive - dominance model

A,B,C-Scales used to test the presence/absence of epistasis; [m]-mean;[d]-additive;[h]-dominant;[i] axa; [j]-axd; [l]-dxd

In the crosses showing non-allelic interactions as revealed by the significance of the scale, the additive component [d] was significantly positive for days to first flowering, days to maturity and plant height in TMV 4 x KS 99037; plant height and number of branches in TMV 5 x KS 990813; days to first flowering and days to maturity in the cross TMV $5 \mathrm{x}$ KS 99153; indicating the presence of additive gene action. None of the crosses exhibited additive genetic variance for the traits capsule length and oil content. Variances observed in the above crosses for the characteristics indicated will be useful in pedigree breeding for selection of superior segregants. The additive gene action for yield and yield related characteristics was reported earlier by many scientists. Sridharan (1992) and Ragiba and Raja Reddy (2000) reported additive genetic variance for days to maturity. Dharmalingam, (1990) reported additive gene action for plant height and number of branches. Additive genetic variance was reported by Saravanan and Nadarajan (2003) for days to 50 per cent flowering and Krishnaiah et al. (2003) for plant height.

The dominance component [h] was positive and significant for days to first flowering, days to maturity, plant height, number of capsules, hundred seed weight and oil content in the cross TMV 4 x KS 99037; all the other characteristics except number of branches and oil content in TMV5 x KS 99153; number of capsules, capsule length, number of seeds per capsule and seed yield in TMV4 $x$ KS99153; number of capsules, seed yield and oil content in TMV5 x KS99037; capsule length, number of seeds per capsule and oil content in TMV4 x KS990813 and number of seeds per capsule in TMV5 x KS990813 indicating the presence of dominance gene action for the above mentioned characteristics in the respective crosses. Dominance gene action was reported by Ramesh et al. (2000) for days to 50 per cent flowering; Sivagamy, (2003) for days to maturity and plant height; Vidhyavathi (2002) for number of branches; Saravanan and Nadarajan (2003) for number of capsules, number of seeds per capsule and seed yield per plant.

As the characteristics indicated above are governed mainly by dominance gene action, relevant crosses may not be amenable for improvement by simple selection techniques. Combining the stabilized genotypes resulting from those crosses, in a planned way for exploiting heterosis directly in hybrids would be possible.

Both additive and dominance gene actions were found to be present in the crosses TMV 4 x KS 99037 and, TMV 5 x KS 990813 for days to first flowering; TMV 4 x KS 99037 and TMV 5 x KS 990813 for days to maturity; TMV 4 x KS 99037 for plant height. The simultaneous occurrence of the additive and dominance gene action makes it necessary for resorting to special techniques like intermating segregants or recurrent selection to harness the different kinds of gene effects. The predominance of both additive and dominance gene effects was also reported by Pathak and 
Dixit (1988) for capsules per plant and Dharmalingam (1990) for number of seeds per capsule in eight out of 15 combinations.

The additive $\mathrm{x}$ additive gene action was the only fixable epistatic component of genetic interaction observed in the crosses, TMV $4 \mathrm{x}$ KS 99037 for all the characteristics except capsule length and number of seeds per capsule; TMV 5 x KS 99153 for all other characteristic except for number of primary branches and oil content; TMV 5 x KS 99037 for number of capsules, 100 seed weight and seed yield; TMV 4 x KS 99153 for capsule length and number of seeds per capsule; TMV 5 x KS 990813 for capsule length and number of seeds per capsule; TMV 4 x KS 990813 for number of seeds per capsule and oil content. Additivity can be fixed by simple pedigree selection for the improvement of the respective characteristics in the above crosses. The prevalence of additive $\mathrm{x}$ additive epistasis was earlier reported by Dharmalingam, (1990) for number of branches per plant, number of capsules on main stem, length of capsule, 100-seed weight and seed yield per plant in most of the crosses.

The opposite signs of [h] and [1] components were revealed by the crosses TMV4 x KS 99037 for eight characteristics, TMV4 x KS 990813 for seven characteristics, TMV 4 x KS 99153 for six characteristics, TMV $5 \mathrm{x}$ KS 99037 seven characteristics, TMV 5 x KS 990813 for seven characteristics, TMV $5 \mathrm{x}$ KS 99153 for eight characteristics, indicating the predominance of duplicate epistasis in the above crosses in respect of their economic characteristics. However, the complementary epistasis was evident for number of capsules per plant and seed yield in TMV $4 \times \mathrm{KS}$ 990813, oil content in TMV 4 x KS 99153, number of primary branches, capsule length and oil content in TMV 5 x KS 99037, number of primary branches in TMV $5 \mathrm{x} \mathrm{KS}$ 990813, as noticed from the same sings of [h] and [1] components. In general duplicate epistasis was found to be predominant when com- pared to complementary epistasis. Therefore, in order to exploit the presence of interaction effects mainly dominance $\mathrm{x}$ dominance and duplicate epistasis $\mathrm{x}$ dominance type, the delayed selection and intermating of segregants followed by recurrent selection will be helpful for improvement. Sridharan, (1992) observed duplicate epistasis for seed yield. Deenamani (1989) reported duplicate epistasis for days to 50 per cent flowering, plant height, number of branches, number of capsules, number of seeds per capsule and seed yield. Dharmalingam, (1990) and Thirugnanakumar, (1991) also observed duplicate epistasis for different economic attributes. Additive as well as additive $\mathrm{x}$ additive interaction effects along with duplicate epistatis was observed in the crosses TMV 4 x KS 99037 for days to first flowering, days to maturity and plant height; TMV 5 $\mathrm{x}$ KS 99153 for days to first flowering and days to maturity. Rapid improvement by simple selection programme is possible, as the transgressive segregants available in this cross may get stabilized in the early generations by simple pedigree breeding procedures.

\section{Inheritance studies}

Inheritance of branching was studied with $\mathrm{F}_{2}$, $\mathrm{BC}_{1}$ and $\mathrm{BC}_{2}$ generations of the crosses with branched and monostem / shy branching accessions (Table 3). Among the six crosses studied, five crosses viz., TMV 4 x KS 99037, TMV 4 x KS 990813, TMV 5 x KS 99037, TMV 5 x KS990813 and TMV 5 x KS99153, showed a monogenic inheritance of 3:1 with simple dominance recessive relationship for branching and monostem / shy branching characteristics respectively. Brar and Ahuja (1979) reported that the branched $(\mathrm{Nb})$ and nonbranched (nb) traits are monogenically controlled and non-branched trait is controlled, by recessive genes. Baydar and Turgut (2000) also reported 3: 1 segregation ratio for branching and non-branching types, respectively. The cross TMV 4 x KS 99153 displayed a segregation ratio of 15:1 for the pres- 
ence of branched and monostem / shy branching characteristics respectively indicating the presence of duplicate dominant gene action. Generation mean analysis also revealed duplicate epistasis for number of branches in this particular cross.

The segregation for $\mathrm{BC}_{1}$ progenies was expected to be in the ratio of 2:0 and 4:0 for branching and monostem / shy branching characteristics for monogenic and digenic $\mathrm{F}_{2}$ progenies. This cross also showed duplicate epistasis for the number of branches per plant in generation mean analysis. Such coincidence of genetic and biometric results although rare could be of great value in improving the trait, number of branches per plant. In all the other crosses the observed test cross segregation could not fit with the expected ratios.

Table 3 . Chi square test for goodness of fit with standard ratios in the segregating generations for monostem / shy branching

\begin{tabular}{|c|c|c|c|c|c|}
\hline \multirow{2}{*}{$\begin{array}{l}\text { Cross } \\
\text { Combination }\end{array}$} & \multirow[b]{2}{*}{$\begin{array}{c}\text { Gen- } \\
\text { eration }\end{array}$} & \multicolumn{2}{|c|}{ Expected ratio and observed segregation } & \multirow[b]{2}{*}{$c^{2}$ value } & \multirow[b]{2}{*}{ Remarks } \\
\hline & & $\begin{array}{l}\text { Expected ratio } \\
\text { (Branch: Monostem) }\end{array}$ & Observed & & \\
\hline \multirow{4}{*}{ TMV 4 x KS99037 } & $\overline{\mathrm{F}_{2}}$ & - & $3: 1$ & 1.44 & Non significant \\
\hline & $\mathrm{BC}_{1}$ & $2: 0(1: 1)$ & - & - & - \\
\hline & $\mathrm{BC}_{2}$ & $1: 1$ & - & - & - \\
\hline & $\mathrm{F}_{2}$ & - & $3: 1$ & 0.19 & Non significant \\
\hline \multirow[b]{2}{*}{ TMV 4 x KS990813 } & $\mathrm{BC}_{1}$ & $2: 0(1: 1)$ & - & - & - \\
\hline & $\mathrm{BC}_{2}$ & $1: 1$ & - & - & - \\
\hline \multirow{4}{*}{ TMV 4 x KS99153 } & $\mathrm{F}_{2}$ & - & $15: 1$ & 0.21 & Non significant \\
\hline & $\mathrm{BC}_{1}$ & $4: 0(1: 1)$ & - & - & - \\
\hline & $\mathrm{BC}_{2}$ & $3: 1$ & $3: 1$ & 0.19 & Non significant \\
\hline & $\mathrm{F}_{2}$ & - & $3: 1$ & 1.32 & Non significant \\
\hline \multirow[b]{2}{*}{ TMV 5 x KS99037 } & $\mathrm{BC}_{1}$ & $2: 0(1: 1)$ & $2: 0$ & 0.00 & Non significant \\
\hline & $\mathrm{BC}_{2}$ & $1: 1$ & - & - & - \\
\hline \multirow{5}{*}{ TMV 5 x KS990813 } & $\mathrm{F}_{2}$ & - & $3: 1$ & 0.02 & Non significant \\
\hline & $\mathrm{BC}_{1}$ & $2: 0(1: 1)$ & - & - & - \\
\hline & $\mathrm{BC}_{2}$ & $1: 1$ & - & - & - \\
\hline & $\mathrm{F}_{2}$ & - & $3: 1$ & 3.61 & Non significant \\
\hline & $\mathrm{BC}_{1}$ & $2: 0(1: 1)$ & - & - & - \\
\hline TMV 5 x KS99153 & $\mathrm{BC}_{2}$ & $1: 1$ & $1: 1$ & 1.88 & Non significant \\
\hline
\end{tabular}

ratios respectively. This ratio was fitted well with only one cross TMV 5 x KS 99037 with the monogenic $\mathrm{F}_{2}$ ratio of $3: 1$. In general the ratio observed for $\mathrm{BC}_{1}$ progenies was $3: 1$ for branched and monostem / shy branching habits in most of the crosses. This variation may be due to the effect of modifier genes. In the test cross progenies $\left(\mathrm{BC}_{2}\right)$ the segregation ratios differed according to the observed epistatic ratios. The segregants in the test cross progenies fitted well with the expected ratio of 1:1 in the cross TMV $5 \times$ KS 99153 which was expected to have a $3: 1 \mathrm{~F}_{2}$ monogenic ratio. This cross showed adequacy for the additive dominance model through generation mean analysis. The cross TMV4 $\mathrm{x}$ KS 99153 which showed 15:1 $\mathrm{F}_{2}$ ratio, fitted to the expected ratio of $3: 1$ of the test cross
Thus, a complexity of inheritance due to the interaction of modifying genes, which could alter the segregation pattern, was observed. It is suggested, from the above study, that the hybridization followed by pedigree selection would be of great value for exploitation of additive and non - additive variances for number of branches and other yield related characteristics in sesame improvement.

\section{CONCLUSIONS}

The study concluded that the additive, dominance and duplicate dominance gene effects were found to be important for number of branches per plant. Additive $\mathrm{x}$ additive fixable gene interaction was also reported in different crosses. In most of the crosses, duplicate epis- 
tasis was found to be predominant when compared to complementary epistasis. Thus gene effects for a given characteristics varied among crosses. Inheritance study showed a monogenic inheritance of 3:1 with simple dominance recessive relationship for branching and monostem / shy branching characteristic, respectively. Further, detailed study on this characteristic will help to develop a mono stem or shy branching variety of sesame suited to high - density planting, which will benefit the farming community by increased net return.

\section{REFERENCES}

Baydar, H. and Turgut, I. (2000). Studies on genetics and breeding of sesame (Sesame indicum L ). I. Inheritance of the characters determining the plant type. Turkish journal of Biology. 24 : 503-512.

Brar, G.S. and Ahuja K.L. (1979). Sesame: its culture, genetics, breeding and biochemistry. In: Malik, C.P. (ed.). Ann. Rev. of Plant Sci. Kalyani publishers, New Delhi. p. 245-313.

Cavalli, L.L. (1952). An analyses of linkage in quantitative inheritance. In: Quantitative inheritance (Reeve, R.C.R. and C.H. Waddington, Eds.), HMSD, London. 135 $-144$.

Deenamani, I.E.S.K.(1989). Genetic architecture of yield and yield components in sesame. M.Sc.(Ag.) Thesis, Tamil Nadu Agricultural University, Coimbatore, India.

Dharmalingam, V. (1990). Genetic basis of yield and its components for crop improvement in Sesame indicum L. Ph.d., Thesis, Tamil Nadu Agricultural University, Coimbatore, India

Hayman, B.I. 1958. The separation of epistasis from additive and dominance variation in generation means. Heredity. 12 : 371390.

Jinks, J. L. and Jones R.M. 1958. Estimation of the components of heterosis. Genetics, 43: $223-234$.
Joshi, A.B. 1961. Sesamum. Pub. The Indian Central oilseeds committee. Hyderabad.

Krishnaiah, G., Rajareddy, K. and Reddy Sekhar, 2003. Heterosis and Combining ability in sesame. J. Oilseeds Res., 20 : 229- 233.

Lal, J.P. and Rai B. 1991. Sesamum: An all season oilseed crop. Indian farming. 41: 24-27.

Mather, K. and Jinks J.L. 1971. Biometrical Genetics - The study of continuous variation. Chapman and Hall Ltd., London.

Pathak, H.C. and Dixit S.K..1988. Genetic analysis for single stemmed sesame (Sesamum indicum L.). Indian J. Genet., $48: 325-330$.

Ragiba, M. and Raja Reddy C. 2000. Combining ability in a diallel cross of sesamum (Sesamum indicum L.). Ann. Agric. Res., $21: 123-128$.

Ramesh, S., Sherif, R. A., Mohan Rao, A. and Lalitha Reddy, S. S. 2000. Line x tester Analysis of quantitative traits in sesame (Sesamum indicum L.) Mysore J. agric. Sci. 34: 308-310.

Saravanan, S. and Nadarajan, N.2003. Combining ability studies in sesame. Crop Res. 25(2) : $319-324$.

Sivagamy, 2003. Combining ability, heterosis for yield and its attributes in sesame (Sesamum indicum L.). M.Sc.(Ag.) Thesis, Tamil Nadu Agricultural University, Coimbatore, India.

Sridharan, C. S. 1992. Study of grne action for seed yield and related traits in sesame (Sesamum indicum L.) by generation mean and triple test cross analysis. Ph.D. Thesis, Tamil Nadu Agricultural University, Coimbatore, India.

Thirugnanakumar, S. 1991. Seed genetics in relation to yield in sesame (Sesamum indicum L.). Ph.D. Thesis, Tamil Nadu Agricultural University, Coimbatore, India.

Vidhyavathi, 2002. Study of heterosis and combining ability in sesame (Sesamum 
indicum L.) M.Sc.(Ag.) Thesis, Tamil Nadu Agricultural University, Coimbatore, India.

Weiss, E.A. 1971. Castor, sesame, and safflower. Leonard Hill Books, London. p. 311-525.

Weiss, E.A. 1983. Oilseed crops. Longman, London. p. 282-340. 